\title{
An integration of perspectives on aging and consumer decision making
}

\author{
Catherine A. Cole ${ }^{\mathrm{a}, *, 1}$, Michelle P. Lee ${ }^{\mathrm{b}, 1,2}$, Carolyn Yoon ${ }^{\mathrm{c}, 1,3}$ \\ ${ }^{a}$ Henry B. Tippie College of Business, University of Iowa, 108 PBB, S248, Iowa City, IA 52242-1000, USA \\ ${ }^{\mathrm{b}}$ Lee Kong Chian School of Business, Singapore Management University, 50 Stamford Road, Singapore 178899, Singapore \\ ${ }^{\mathrm{c}}$ Stephen M. Ross School of Business, University of Michigan, 701 Tappan St., Ann Arbor, MI 48109-1234, USA
}

Available online 29 January 2009

\begin{abstract}
In this response we integrate the three Comments (Goldberg, forthcoming; Healey \& Hasher, forthcoming; Sternthal \& Bonezzi, forthcoming) on our original article (Yoon, Cole, \& Lee, forthcoming) and suggest both enhancements to our conceptual framework and future research directions. As a group, the responses help guide future research by ably highlighting certain complexities of studying consumer decision making and aging. Acknowledging these complexities and nuances, while encouraging research on the effects of age on consumer decision making, should help spark a new wave of studies in the area.
\end{abstract}

(C) 2008 Society for Consumer Psychology. Published by Elsevier Inc. All rights reserved.

Participating in the Research Dialogue section of $J C P$ has been educational and stimulating. The suggestions and comments from the three groups of commentators were especially intriguing in that they approach consumer decision making and aging from very different angles, thereby adding to the richness of the discussion. In this response, we integrate the three comments and our original article to suggest enhancements to our conceptual framework as well as future research directions.

\section{Strategies to increase fit}

The conceptual framework in Yoon, Cole, and Lee (forthcoming) identified strategies that consumers might use when situations of low fit occur, and were referred to as consumer adaptations. Sternthal and Bonezzi's (forthcoming) discussion of accommodation and stimulating self-regulation, on the other hand, offers a somewhat different perspective by considering what can be done to ensure a greater fit in the first place. Accommodation strategies are largely ways to decrease the demands of the task to match the cognitive resources available to older consumers, or to match their preferences or tendencies in processing style. Stimulating self-regulation, by not presuming that apparent declines in older adults' cognitive functions

\footnotetext{
* Corresponding author. Fax: +1 3193353690.

E-mail addresses: cathy-cole@uiowa.edu (C.A. Cole), michlee@smu.edu.sg (M.P. Lee), yoonc@umich.edu (C. Yoon).

${ }^{1}$ Authors are listed alphabetically.

${ }^{2}$ Fax: +65 68220777.

${ }^{3}$ Fax: +1 7349368716.
}

need to be accommodated, takes the opposite tack, and identifies the stimulation of elaboration when processing information as a means of pre-empting occurrences of low fit.

With respect to the latter, the authors argue that it is the failure to elaborate on message information that accounts for particularly poor performance among the elderly, especially in the processing of communications with an assertion-plus-tag structure. We concur with the authors, but add that the argument might be couched too broadly as a failure to elaborate. Older adults have been shown to have diminished inhibition in terms of suppressing irrelevant thoughts and information (Hasher \& Zacks, 1988). Arbuckle and Gold (1993), for instance, found that age-related declines in ability to inhibit task-irrelevant information account for the greater verbosity among older adults, with a characteristic lack of focus.

It seems the issue might be better described as a need for more directed elaboration. The finding that simply asking older adults to think deeply did not prompt detailed processing (Cole \& Houston, 1987) while more directed instructions to form a mental image (Law, Hawkins, \& Craik, 1998) or to justify a decision (Kim, Goldstein, Hasher, \& Zacks, 2005) led to enhanced performance, supports that contention. Perhaps older individuals are not sufficiently task-oriented in their elaboration, or they may be less able to inhibit irrelevant thoughts that can intrude in the course of elaboration. Similarly, the elderly's deficiency in processing communications with assertion-plus-tag structures is not a failure to elaborate per se, but a failure to engage in a particular type of elaboration - that which facilitates the integration of negators and source information with the assertion. The challenge to researchers may therefore be to identify the types of elaboration at 
which older adults are proficient or deficient relative to younger adults and to better understand how a better matching of elaboration type and task requirements might be brought about.

Goldberg (forthcoming), like Sternthal and Bonezzi (forthcoming), begins with the premise that older consumers face cognitive deficits that need to be addressed, but approaches the issue from the perspective of interventions that may be undertaken in the areas of law, consumer education and marketing. Among the marketing interventions mentioned are ones that would appropriately be termed strategies of accommodation, as described by Sternthal and Bonezzi (forthcoming), such as high-tech devices that help with making nutritional food choices and medical monitoring devises. Other measures, such as cardiovascular fitness training, cognitive training and building social connectedness, are akin to Sternthal and Bonezzi's notion of stimulating self-regulation, in that they are aimed at improving the cognitive readiness of older consumers so that there is a greater match between resources and task demands.

\section{Bases for optimism}

Healey and Hasher's (forthcoming) comments take an altogether different perspective by presenting a compelling cautionary argument against overemphasizing cognitive deficits in the elderly at the risk of overlooking (1) cognitive processes that are age-invariant, (2) instances where cognitive impairments that accompany aging can, in fact, improve decision quality, and (3) instances where what may seem like a cognitive impairment on one task can be beneficial on another.

This perspective raises a number of interesting research questions. Granting that some cognitive processes decline with age while others are age-invariant, what practical significance is there in terms of the dependence of day-to-day consumer tasks on age-dependent versus age-invariant cognitive processes? For instance, given that the abilities to judge frequency (Hasher \& Zacks, 1988) and detect correlations (e.g. Kareev, Liberman, \& Lev, 1997) appear to be preserved with age, do such abilities account for a significant enough proportion of consumer decisions to ease concerns about the negative impact of aging? Also, on any given task, can the same decision quality or level of satisfaction be achieved by relying alternatively on age-invariant functions rather than on more age-dependent ones? If so, what can be done from public policy and managerial perspectives to encourage a more spontaneous invocation of age-invariant functions in decision making by older consumers? Finally, if some cognitive constraints can indeed lead to better judgment and decision making, such as when certain heuristics are used, can they be leveraged by encouraging a reliance on those heuristics?

Healey and Hasher (forthcoming) make an interesting point by highlighting that what may seem disadvantageous to older adults might in fact be beneficial, such as how the inability to inhibit irrelevant information might prove to be advantageous when that information is unexpectedly called for. Similarly, while the fluency heuristic can lead the elderly astray when relied upon in making judgments of truth (Skurnik, Yoon, Park, \& Schwarz, 2005; for a review see Schwarz, Sanna, Skurnik, \& Yoon, 2007), it might, in other situations, be reliably used for accurate performance, such as in recognition judgments. Since some supposed 'impairments' can cut both ways in terms of helping or hindering performance, it would be useful to more thoroughly investigate what those 'impairments' are and the conditions under which they might be beneficial to the task, so that they might better inform public policy and marketing decisions.

\section{A Nuanced relationship between cognitive processes and decision making}

Overall, we concur with Healey and Hasher (forthcoming) that it is better to acknowledge that the effects of cognitive changes are nuanced; they can be negative, neutral or positive. We add here that the effects may also be nonlinear or even reversed (Goldberg, forthcoming). For example, a study by Cole and Balasubramanian (1993) found that verbal working memory does not mediate age effects on search intensity, but that those consumers with very low levels of working memory search less intensely. This pattern of results suggests that ageassociated changes in verbal working memory do not affect search intensity unless working memory falls below a very low level. It also illustrates the relationship between a cognitive process and a decision strategy should not be presumed linear, or even monotonic, across their relevant ranges. With respect to the plasticity of cognitive changes, Goldberg (forthcoming) noted the failure of government and education in helping older adults deal with cognitive change and the contrary successful efforts of both for-profit and social marketers in reversing cognitive changes in the older population through cardiovascular fitness and cognitive training programs.

\section{Construal level theory as a basis for understanding performance of older consumers}

Sternthal and Bonezzi's (forthcoming) suggestion that we incorporate construal level theory (Liberman, Trope, \& Wakslak, 2007) in our model of aging is noteworthy. The argument presented is that older people are more likely to adopt high-level construals that stimulate a focus on abstract self-regulatory goals (e.g. living a healthy life style). Individuals who use more abstract goals construe stimuli with relatively simple representations that extract the gist from available information (Kim \& Roedder John, 2008) so that they are influenced by abstract and general features of the stimuli, such as quality (Dhar \& Kim, 2007). In contrast, people who use low-level construals construct concrete mental models of stimuli with relatively complex representations and may be influenced by specific attributes, such as price. So, one strategy to enhance performance on decision making among older consumers is to make sure the higher-order goals are relevant for the task. Another strategy is to develop decision aids that prompt a step-by step decision making process typical of the use of low level-construals.

\section{An integration of perspectives}

The conceptual framework presented in Yoon et al. (forthcoming) detailed consumer adaptations enabling older 
consumers to cope with decision making in situations of low fit. The comments of Sternthal and Bonezzi's (forthcoming) and of Healey and Hasher (forthcoming) have brought into focus a missing component of this framework: strategies that enhance fit or prevent situations of low fit from occurring in the first place. Among these strategies are (1) those that reduce the demands of the task to accommodate the elderly's resource availability, (2) those that serve to stimulate what may be considered latent resources so that the resource demands of the task are met, and (3) those that leverage cognitive functions that are age-invariant or that have beneficial outcomes arising from what may seem like cognitive deficits.

Goldberg's (forthcoming) comments have also underscored both the need to ensure greater fit and the need to mitigate the detrimental impact of low fit from the perspectives of law, consumer education, and marketing. More importantly, his analysis concerning the limited effectiveness of current legal and consumer education interventions suggests that improvements may perhaps be gained with a greater leveraging of knowledge gleaned from aging research.

\section{Consumer decision making: internal and external validity}

These three commentaries highlight underlying tensions among what might be termed basic research, consumer decision making research, and managerially relevant decision research. In our original article we sidestepped these issues by adopting a broad definition of consumer decision making, one that includes the processes and outcomes related to everyday problem solving in consumer domains. For example, imagine a consumer deciding about a treatment option for a specific type of cancer, or selecting a Medicare prescription drug plan. These decisions will involve a complex set of judgments and memory tasks, including frequency and probability judgments, assessments about correlations between prices and quality, and use of both working and long-term memory.

A tradition in the psychology literature is to study the building blocks of decision making in order to identify basic principles. In this tradition, internal validity is critical. In consumer psychology, however, researchers need to consider decision making in the sorts of complex environments that consumers actually face. Consequently, consumer researchers often struggle to identify tasks that are externally valid. Yet another source of difficulty is one intrinsic to the marketing literature itself, where a main goal is managerial relevance.

Clearly there is a resolution, one that involves multiple studies, some aimed at ensuring internal validity occurring at the basic level in a laboratory setting, and others focusing on external validity by conducting field studies that apply these basic level insights to general decision making. Shadish (2002), for example, notes that medicine has greatly advanced knowledge using field study methodology, despite such well-known shortcomings as selection bias, attrition, and problems of implementation and statistical analysis. Nonetheless, if consumer psychologists wish to design interventions to improve decision making among either group (younger or older consumers), then a combination of laboratory and field studies needs to be undertaken.

\section{Conclusion}

We write this response feeling more optimistic and positive about aging than when we wrote the original article, in that we now recognize that increasingly aging and decision making is a burgeoning research area with many interesting questions that need to be addressed. Our hope is that other researchers will share in our enthusiasm and will pursue research programs that seek to develop a better understanding of aging and consumer decision making.

\section{References}

Arbuckle, T. Y., \& Gold, D. P. (1993). Aging, inhibition and verbosity. Journal of Gerontology, 48(5), 225-232.

Cole, C. A., \& Balasubramanian, S. K. (1993). Age differences in consumers' search for information: Public policy implications. Journal of Consumer Research, 20(1), 157-169.

Cole, C. A., \& Houston, M. J. (1987). Encoding and media effects on consumer learning deficiencies in the elderly. Journal of Marketing Research, 24(1), 55-63.

Dhar, R., \& Kim, E. Y. (2007). Seeing the forest or the trees: Implications of construal level theory for consumer choice. Journal of Consumer Psychology, 17(2), 96-100.

Goldberg, M.E. (forthcoming), "Consumer decision making and aging: Current knowledge and future directions: A commentary from a public policy/ marketing perspective,". Journal of Consumer Psychology.

Hasher, L., \& Zacks, R. T. (1988). Inhibitory mechanisms and the control of attention. In A. Conway, C. Jarrold, M. Kane, A. Miyake, \& J. Towse (Eds.) Variation in working memory : Oxford University Press.

Healey, K. M. \& Hasher, L. (forthcoming), "Limitations to the deficit attenuation hypothesis: Aging and decision making,". Journal of Consumer Psychology.

Kareev, Y., Lieberman, I., \& Lev, M. (1997). Through a narrow window: Sample size and the perception of correlation. Journal of Experimental Psychology: General, 126(3), 278-287.

Kim, S., Goldstein, D., Hasher, L., \& Zacks, R. T. (2005). Framing effects in younger and older adults. Journals of Gerontology: Psychological Sciences and Social Sciences, 60B(5), 215-218.

Kim, H., \& Deborah, R. J. (2008). Consumer response to brand extensions: Construal level as a moderator of the importance of perceived fit. Journal of Consumer Psychology, 18, 116-126 (April).

Law, S., Hawkins, S. A., \& Craik, F. I. M., (1998). "Repetition-induced belief in the elderly: Rehabilitating age-related memory deficits,". Journal of Consumer Research, 25(2), 91-107.

Liberman, N., Trope, Y., \& Wakslak, C. (2007). Construal level theory and consumer behavior. Journal of Consumer Psychology, 17, 113-117.

Schwarz, N., Sanna, L., Skurnik, I., \& Yoon, C. (2007). Metacognitive experiences and the intricacies of setting people straight: Implications for debiasing and public information campaigns. Advances in Experimental Social Psychology, 39, 127-161.

Shadish, W. R. (2002). "Revisiting field experiments: Field notes for the future,". Psychological Methods, 7(1), 3-18.

Skurnik, I., Yoon, C., Park, D. C., \& Schwarz, N. (2005). "How warnings about false claims become recommendations,". Journal of Consumer Research, 31(4), 713-724.

Sternthal, B., \& Bonezzi, A. (forthcoming), "Comments on Yoon, Cole and Lee: Consumer decision making and aging: Current knowledge and future directions,". Journal of Consumer Psychology.

Yoon, C., Cole, C. A., and Lee, M. P. (forthcoming), Consumer decision making and aging: Current knowledge and future directions,". Journal of Consumer Psychology. 